\title{
DOA Estimation-a Comparative Analysis
}

\author{
Ayesha Naaz and Rameshwar Rao
}

\begin{abstract}
In this paper, the direction of arrival (DOA) angle estimation of signals impinging on 3- $D$ array of sensors in cubical arrangement is studied. The results thus obtainedwere compared with the direction of arrivals obtained with a combination of two uniform square arrays which were considered in parallel to form a structure as cube. MUSIC algorithm (Multiple Signal Classification) was used to estimate the directions of arrival (DOA) of the signals .Also in this paper cubical array geometry for low signal to noise ratio was tested and the results compared were with that of the two parallel square arrays .Experimental results demonstrate that the cubical geometry has better detection capability as compared to two 2-D square arrays with the same or even a higher SNR.
\end{abstract}

Index Terms-Array processing, direction of arrival, MUSIC, 3-D array.

\section{INTRODUCTION}

Array signal processing uses an array of sensors or antennas to locate the signal and to determine information about them. Modern array signal processing applications place great demand on the underlying array geometry to deliver high performance.

The problem of estimating the Direction of arrival (DOA) of sources via an array of sensors is a major research issue in tomography, seismology, sonar, radar, communication. The objective is to extract useful characteristics from the received signal field (i. e, the number of signals, the signal directions, the signal strengths, etc.).In signal processing, Direction of Arrivalinvolves estimation of azimuth and elevation angles of the propagating waveforms which is very crucial and has many applications in various fields of engineering. Many high resolution DOA estimation methods, such as famous MUSIC and ESPRIT have been developed [1]. Various other methods include the root MUSIC, Matrix Pencil method, Multiple Invariance ESPRIT, Subspace fitting method.

The ability of achieving high performance is greatly influenced by the geometry of the array. There are basically three types of array geometries i.e., One Dimensional (1 D), Two Dimensional (2 D) and Three Dimensional (3 D). The $1 \mathrm{D}$ array geometry where usually a set of sensors are uniformly spaced in a line generally termed as a uniform linear array (ULA), have been dealt in [2]. [3] proposed a new algorithm for wide band sources impinging on ULA. In 2 D geometry, uniform rectangular/square arrays were investigated in [4],Uniform Circular arrays in [5] and [6], L

Manuscript received August 4, 2013; revised January 25, 2014.

Ayesha Naaz is with Department of Electronics and Communication Engineering at MuffakhamJah College of Engineering and Technology, Hyderabad, India ( e-mail: ayeshanaaz10@gmail.com).

Rameshwar Rao is with Jawarharlal Nehru Technological University, Hyderabad, India (e-mail: rameshwar_rao@hotmail.com). shape arrays in [7] and [8], triangular arrays were considered in [9] and [10]. For the 3 dimensional case sphere has been investigated using Matrix Pencil Method in [6] and [10]. Of the most popular direction finding methods MUSIC (Multiple Signal Classification) has received most attention as it can be applied to any arbitrary geometry but ESPRIT (Estimation of Signal Parameters via Rotational Invariance Technique) is limited to geometry with identical pair of sensors.

In this paper, a cubical array (3 D geometry) with MUSIC algorithm is investigated for determining azimuth and elevation angles of signals with different SNR values. The results were compared with the results obtained by considering two uniform square arrays arranged in parallel with the same spacing as is the distance between the sensors in the square array. One dimensional array suffers from 'East-West ambiguity' and 'north-south ambiguity'. In two Dimensional arrays east west ambiguity is removed but north south ambiguity still remains.

The limitations of the one dimensional and two dimensional arrays create problems for locating sources with much accuracy. The array's design is highly extensible, however, and it is an important building block for more complex three dimensional arrays such as a pyramid, cube, and cylinder, cone which uses multiple linear arrays or more exotic shapes such as circle.

The following assumptions were made on the signal models which are considered throughout this paper.

Assumption 1: The signals emitted from the sources are narrow band random process all with the same frequency and stationary stochastic signal $s(t)$.

Assumption 2: The radiating sources are located in far fields of the array. This enables us to model the waves impinging on the array as plane waves.

Assumption 3: The array and the sources are confined to a plane. This enables us to associate each source with a single angle $\theta$ for the $k^{\text {th }}$ source, thus the direction of arrival of plane waves with respect to some reference point.

Assumption 4: All the sensors are identical and are all Omni-directional with gain of 1 .

The organization of the paper is as follows: Section II gives the evaluation algorithm (MUSIC) used to find the direction of arrival. Section III depicts the signal model used and Section IV gives the simulation results obtained using MATLAB as the software. Finally the conclusions are presented in Section V.

\section{Music ALGORITHM}

Of the high resolution direction finding algorithms MUSIC algorithm is the one which can be applied to any arbitrary geometry [11]. This is based on eigen decomposition of the signal, also there exists techniques 
without eigen decomposition [12]. Here a cubical geometry is considered to which the MUSIC algorithm which is as given below is applied:

If there are $D$ signals incident onto the array, the received input data vector at an $M$ element array can be expressed as a linear combination of the $D$ incident waveforms and noises.

If $r(t)$ is the array element received signal, $s(t)$ is the source signal, $n(t)$ is additive noise, the first array element is taken as reference array element, then receive signal at the $k t h$ array element is:

$$
r_{i}(t)=\sum_{k=1}^{D} a\left(\theta_{k}\right) s_{k}(t)+n_{i}(t)
$$

where, $i=1,2, \ldots M$

The vector form of which can be written as

$$
r(t)=A s(t)+n(t)
$$

where $r(t)=\left[r_{1}(t), \quad \ldots r_{M}(t)\right]_{M \times 1}^{T}$,

$A$, the matrix of steering vectors is given as

$$
\begin{gathered}
A=\left[\begin{array}{llc}
a\left(\theta_{1}\right) & \cdots & a\left(\theta_{D}\right)
\end{array}\right]_{M \times D}, \\
s(t)=\left[\begin{array}{lll}
s_{1}(t) & \cdots & s_{D}(t)
\end{array}\right]_{D \times 1}^{T} \text { is the signal vector and } n(t)
\end{gathered}
$$$$
=\left[\begin{array}{lll}
n_{1}(t) & \cdots & n_{M}(t)
\end{array}\right]_{M \times 1}^{T} \text { is a noise vector with }
$$
components of variance $\sigma^{2}$.

The received vectors and the steering vectors can be visualized as vectors in an $\mathrm{M}$ dimensional vector space.

The covariance matrix of the array signal vector will be

$$
\begin{aligned}
& R=E\left[r(t) r^{H}(t)\right] \\
& R=A S A^{H}+\sigma^{2} I
\end{aligned}
$$

where $R$ is the signal covariance matrix, $I$ is an identity matrix and $\sigma^{2}$ is the variance of the additive noise.

Let $S$ denote the Covariance matrix of the source signals

$$
S=E\left[s(t) s^{H}(t)\right]
$$

MUSIC requires $S$ be nonsingular, though even that assumption can be relaxed for certain geometries especially the linear uniform array.

Let $\lambda_{1} \geq \lambda_{2} \geq \cdots \geq \lambda_{M}$ and $v_{1} \geq v_{2} \geq \cdots \geq v_{M}$ denote the eigenvalues of $\mathrm{R}$ and A S $A^{H}$ respectively. From the structure of given by (4), these two sets of eigenvalues are related by

$$
\lambda_{i}=v_{i}+\sigma^{2}, i=1,2, \ldots M
$$

Assuming that the matrix $A$ is of full column rank, which is generically the case, it follows that the rank of $A S A^{H}$ is $D$, implying that the $M-D$ smallest eigenvalues of $A S A^{H}$ are equal to zero. Thus

$$
V_{D+1}=\ldots=V_{M}=0
$$

or, equivalently, the smallest eigen value of $R$ is equal to $\sigma^{2}$ with multiplicity $M-D$, i.e.,

$$
\lambda_{D+1}=\cdots=\lambda_{M}=\sigma^{2}
$$

The Eigen values $\lambda_{1}, \ldots, \lambda_{D}$ are known as the signal eigenvalues and $\lambda_{D+1}, \ldots, \lambda_{M}$ as the noise eigen values.

Let $e_{1}, e_{2}, \ldots, e_{M}$ denote the eigenvectors of $R$ correspondingly to $\lambda_{1}, \ldots, \lambda_{M}$.

Form matrices $E_{S}$ and $E_{N}$ as

$$
\begin{aligned}
E_{S} & =\left[\begin{array}{lll}
e_{1} & \ldots & e_{D}
\end{array}\right] \\
E_{N} & =\left[\begin{array}{llll}
e_{D+1} & \ldots & e_{M}
\end{array}\right]
\end{aligned}
$$

$e_{1}, \ldots, e_{D}$ the signal eigenvectors, $e_{D+1}, \ldots, e_{M}$ the noise eigenvectors. $E_{S}$ is the signal subspace and $E_{N}$ is the noise subspace.

By searching through all possible array steering vectors to find those which are orthogonal to the space spanned by the noise Eigen vectors $e_{D+1}, \ldots, e_{M}$ the DOAs $\theta_{1}, \theta_{2}, \ldots, \theta_{p}$ can be determined.

Then $a(\theta)^{*} E_{N} E_{N}{ }^{*} a(\theta)=0$ for $\theta$ corresponding to the DOA of incoming signal. In practice, $a(\theta)$ will not be precisely orthogonal to the noise subspace due to errors in estimating $E_{N}$. However the function

$$
P_{\text {MUSIC }}(\theta)=\frac{1}{a(\theta)^{H} E_{N} E_{N}{ }^{H} a(\theta)}
$$

Known as the multiple signal classification (MUSIC) spectrum can be used to determine the DOA peaks.

This will assume a very large value when $\theta$ is equal to the DOA of one of the signals. The MUSIC algorithm, proposed by Schimdt [1], first estimates a basis for the noise subspace $E_{N}$ and then determines peaks; the associated angles provide the DOA estimates.

The array steering vector, a $(\theta)$ varies according to the geometry of the chosen array which is defined as in the next section.

\section{ARRAY STEERING VECTOR}

A steering vector represents the set of phase delays a plane wave experiences, evaluated at a set of array elements. The phases are specified with respect to an arbitrary origin [13]. For instance, suppose a plane wave is described by a wave vector $k$. If there are $N$ elements in an antenna array, with element ' $i$ ' having location given by

$$
r_{i}=\left(x_{i}, y_{i}, z_{i}\right)
$$

Then the steering vector is an $N \times 1$ complex vector representing the relative phases at each antenna, and is given by

$$
a(\theta)=\left[\begin{array}{c}
e^{j \theta r_{1}} \\
e^{j \theta r_{2}} \\
e^{j \theta r_{3}} \\
\vdots \\
e^{j \theta r_{N}}
\end{array}\right]
$$

The steering vector is written as $a(\theta)$ to make explicit that it depends on the frequency and propagation direction (which determine $\theta$ ) of the plane wave. 
The steering vector for ' $\mathrm{m}$ ' elements is given as:

$$
A(\theta, \varphi)=\left[a\left(\theta_{1}, \varphi_{1}\right), a\left(\theta_{2}, \varphi_{2}\right), \ldots a\left(\theta_{m}, \varphi_{m}\right)\right]
$$

where $\theta_{i}$ and $\varphi_{i}$ represent the azimuth and elevation angles for the signal falling on $i^{\text {th }}$ sensor.

The steering vector for a cubical array was taken by considering the vertices of the cube as shown in Fig. 1.

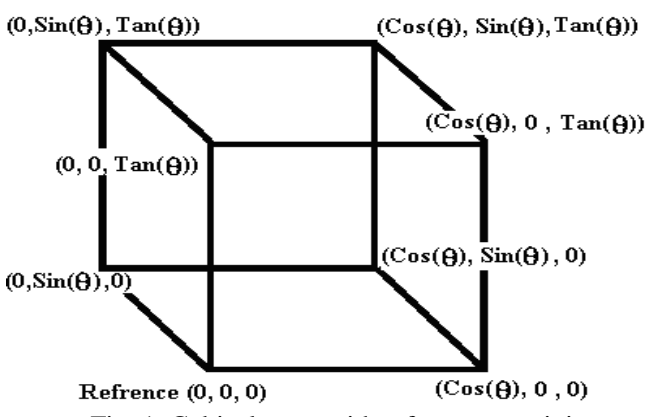

Fig. 1. Cubical array with reference at origin.

The steering vector for one square array was considered with reference at $(0,0,0)$ and the other square array with reference at $(0,0, \tan (\theta))$ was considered as shown in Fig. 2.
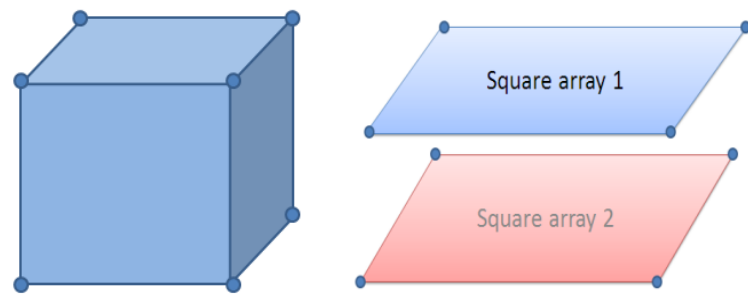

Fig. 2. Acubical array split up as two parallel square arrays.

\section{A. Direction Finding Using Arrays}

The cubical array configuration (8 elements) was used at the receiving terminal and the arriving signals at each sensor were collected and MUSIC algorithm was applied to their co variances.

On similar grounds the cubical array was replaced with two uniform square arrays with same number of elements (4 elements each) and the direction efficiency was tested using MUSIC algorithm and the results of both were compared. [14] Describes the detection of multiple plane waves.

\section{Simulation Results}

In this section the simulation results are presented to demonstrate the efficiency of cubical array as compared to the two square arrays. The simulations were done with code written in MATLAB using MUSIC algorithm for both the arrays. Both the arrays had eight (8) elements and the inter element spacing was taken to be $d=0.5 \lambda$.Three incoming signals with a frequency of $30 \mathrm{KHz}$ were taken and white Gaussian noise was added.

\section{A. Simulations with Two Uniform Square Arrays}

The Fig. 3 gives the results obtained for three incoming which were considered to be arriving at azimuth angles $10^{\circ}, 20^{\circ}$ and $30^{\circ}$ and the elevation angles were considered as $30^{\circ}, 40^{\circ}$ and $80^{\circ}$. The power was estimated as Pmusic 1 for the square array 1 (the array with reference at $(0,0,0)$ and
Pmusic2 for the square array 2 (the array with reference at $(0,0, \tan (\theta))$.

The average of Pmusic1 and Pmusic2 were plotted were plotted. The SNR was considered to be $50 \mathrm{~dB}$.

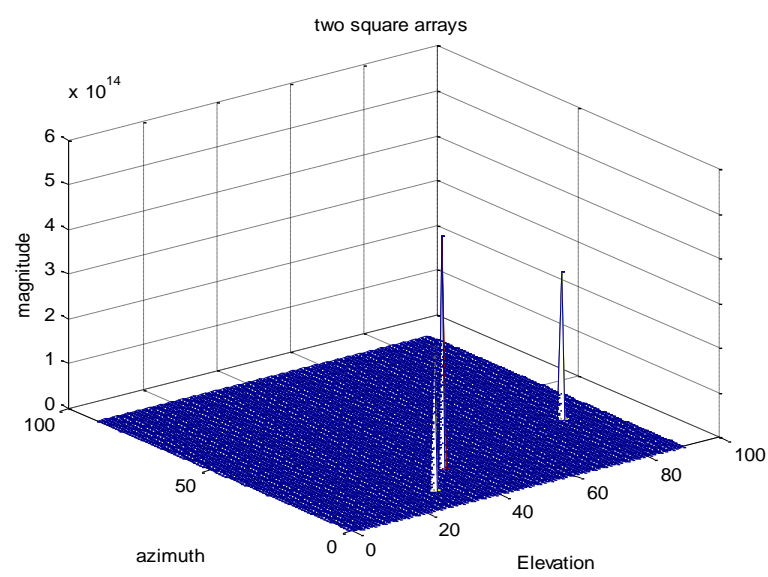

Fig. 3. Spatial spectrum for two Uniform square arrays for $\mathrm{SNR}=50 \mathrm{~dB}$.

The detection of signals by this couple of square arrays was good at $\mathrm{SNR}=50 \mathrm{~dB}$ but when the $\mathrm{SNR}$ was made to $30 \mathrm{~dB}$, the detection ability reduced, which is as shown in Fig. 4.

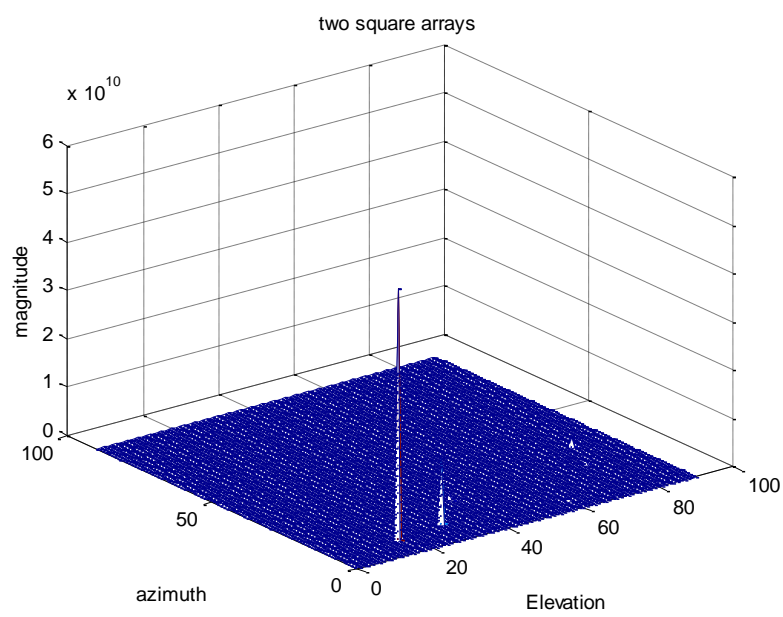

Fig. 4. Spatial spectrum for two Uniform square arrays for $\mathrm{SNR}=30 \mathrm{~dB}$.

\section{B. Simulations with Cubical Array}

The simulations were carried out with a cubical array of eight elements for three signals with $\mathrm{SNR}=50 \mathrm{~dB}$ and $\mathrm{SNR}=30 \mathrm{~dB}$. The results obtained are shown in Fig. 5 and Fig. 6.

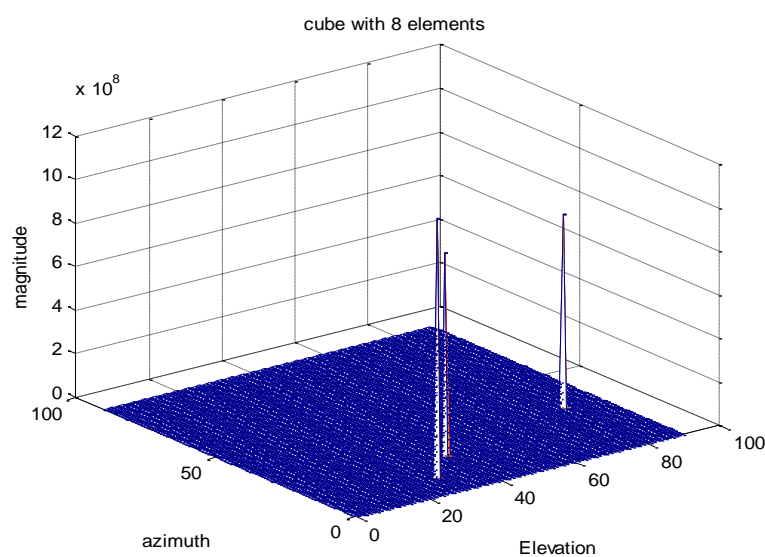

Fig. 5. Spatial spectrum for Cubical array for $\mathrm{SNR}=50 \mathrm{~dB}$. 


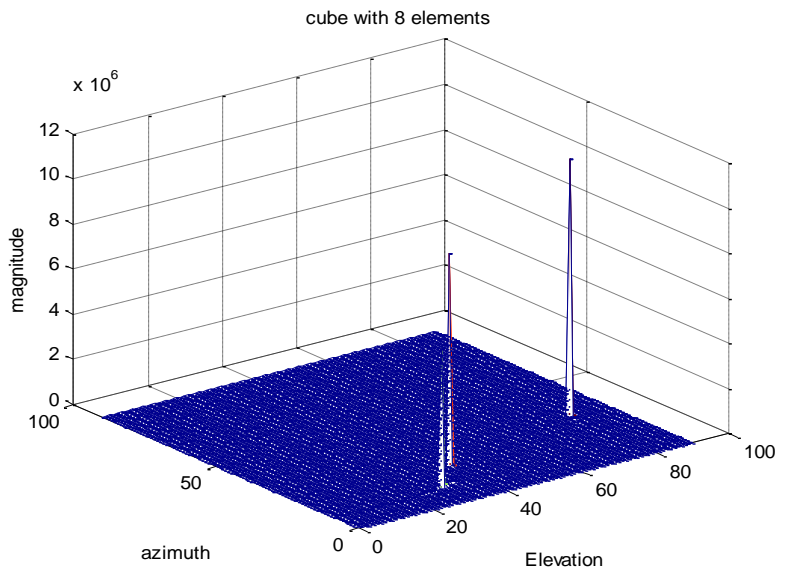

Fig. 6. Spatial spectrum for Cubical array for $\mathrm{SNR}=30 \mathrm{~dB}$.

The cubical array was able to distinguish the two signals clearly and also the peak power was 4 times the peak power obtained in case of square arrays.

\section{CONCLUSIONS}

The paper compares the estimation of direction of arrival of incident signals on a cubical array and a pair of uniform square arrays to form cube. The results show that the 3-D signal at lower SNR as compared to a combination of two uniform square arrays which would be more complex too.square arrays to form cube. The results show that the 3$\mathrm{D}$ array (cubical array) was more efficient in detecting the

\section{REFERENCES}

[1] R. O. Schmidt, "Multiple emitter location and signal parameter estimation," in Proc. the RADC Spectral Estimation Workshop, Rome, NY, 1979, pp. 243-258.

[2] D. H. Xu, J. W. Chen, and Y. Wu, "A novel subspace coherent signal processing algorithm for high resolution DOA estimation," in Proc. ISPACS, 2006.

[3] M. Agrawal and S. Prasad "Estimation of directions of arrival of wideband and wideband spread sources," Signal Processing, vol. 87, issue 4, pp. 614-622, April 2007.
[4] H. Naftali and C. Christodolou, "Uniform circular arrays for smart antennas," IEEE Antennas and Propagation Magazine, vol. 47, no. 4, August 2005.

[5] M. Zeytinoglu, J. litva, and J. Qian, "High resolution direction finding using circular arrays," in Proc. ICASSP, 1991, pp. 3341-3344.

[6] N. Yilmazer and T. K. Sarkar, "2-D unitary matrix pencil method for efficient direction of arrival estimation," Digital Signal Processing, vol. 16 , issue 6 , pp. 767-781, November 2006.

[7] C. Jain, S. X. Wang, and L. Lin "2-D DOA estimation by MEMP based on L shape array," in Proc. ICSP, 2006.

[8] K. T. Wong and M. D. Zoltowski, "High accuracy 2D angle estimation with extended aperture vector sensor arrays," in Proc. ICASSP, 1996, pp. 2789-2792.

[9] S. Lei and M. Sierra-Perez, "Triangular Grid multi beam Array and beam Forming Networks," in Proc. EuCAP 2007. The Second European Conference, 2007, pp. 1-4.

[10] F. Liua, J. Wanga, R. Duc, and G. Yua, "Space-time matrix method for 2-D direction-of-arrival estimation," Signal Processing, vol. 87, pp. 101-106, 2007.

[11] H. L. V. Trees, Optimum Array Processing, New York: Wiley, 2002.

[12] N. Tayem and H. M. Kwon, "Azimuth and elevation angle estimation with no failure and no eigen decomposition," Signal Processing, vol, 86 , issue 1 , pp. 8-16, January 2006

[13] H. Krim and M. Viberg, "Two decades of array signal processing research: the parametric approach," IEEE Signal Process. Mag., vol. 13, no. 4, pp. 67-94, July 1996.

[14] R. Kumaresan and D. W. Tufts, "Estimating the angles of arrival of multiple plane waves," IEEE Trans. AES, vol. 1, p. 134, 1983.

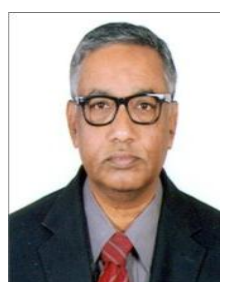

Rameshwar Rao obtained his B.E degree from Osmania University, $\mathrm{M}$ Tech and $\mathrm{Ph} \mathrm{D}$ from IIT Bombay.

$\mathrm{He}$ is currently the vice chancellor of JNTUH and has held various positions of dean of Osmania University, convener PGCET and professor of Department of ECE OU.

His experience spans across 34 years as R \& D engineer and as eminent teacher. His research interests include detection and estimation theory, information and coding theory, VLSI design, VHDL modeling and synthesis.

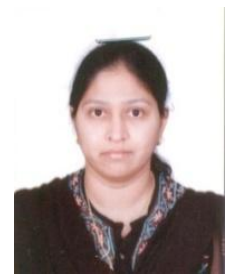

Ayesha Naaz obtained the B.E degree from Osmania University, M. Tech from JNTU Hyderabad and is currently pursuing $\mathrm{PhD}$ in the area of array signal processing. She is currently an associate professor in electronics, she has a teaching experience of 13 years. Her research interests include VHDL modeling and synthesis, signal processing. 